\title{
ADEQUAÇÃO DO SISTEMA DE GERAÇÃO DE VAPOR NA CTE-1*
}

Gustavo Menezes Nevesr ${ }^{1}$
Milson de Andrade Junior
Luis Paulo Silva
Lusco de Souza

\section{Resumo}

Este trabalho tem como objetivo apresentar os resultados do procedimento de adequação do sistema de geração de vapor na CTE-1, visando à redução de custo no grupo CSN. O estudo foi efetuado através da avaliação das condições operacionais dos equipamentos e análise dos processos de geração de energéticos da planta CTE-1, aos quais permitiram diagnosticar as melhores práticas e definir a melhor configuração. Os resultados apresentados foram significativos para as condições de operação atual, o que representa redução do custo para o grupo CSN. Com redução do consumo de vapor e energia.

Palavras-chave: Geração; Vapor; Energia.

\section{Abstract}

\section{STEAM GENERATION SYSTEM ADEQUACY AT CTE1}

This work intends show present the results of the adequacy procedure of the steam generation system in the power plant-1, in order to reduce cost in the CSN group. The study was conducted by evaluating the operating conditions of the equipment and analysis of energy generation processes of power plant-1, which allowed diagnosing better practices and determine the better configuration of the system. The results significant for the current operating conditions, which represents reduction of the cost to the CSN group. By reducing steam and energy consumption.

Keywords: Generation; Steam; Energy.

1 Técnico em Eletrônica, Supervisor de Energéticos, Gerencia de Geração de Energéticos, Companhia Siderúrgica Nacional, Volta Redonda, Rio de Janeiro, Brasil.

2 Engenheiro Eletricista, Engenheiro de Manutenção, Gerencia de Geração de Energéticos, Companhia Siderúrgica Nacional, Volta Redonda, Rio de Janeiro, Brasil.

3 Técnico em Eletrotécnica, Técnico de Desenvolvimento, Gerencia de Geração de Energéticos, Companhia Siderúrgica Nacional, Volta Redonda, Rio de Janeiro, Brasil.

4 Técnico em Eletrônica, Técnico de Desenvolvimento, Gerencia de Geração de Energéticos, Companhia Siderúrgica Nacional, Volta Redonda, Rio de Janeiro, Brasil. 


\section{INTRODUÇÃO}

O Processo de Geração de Energéticos da Central Termoelétrica 1 (1) é composto por três caldeiras (3) constituídas por tubos especiais de aço refratários e três turbo geradores, conforme Figura 1, que fazem a geração de vapor para a produção de energia elétrica de $50 \mathrm{HZ}$, Ar Soprado e Vapor de processo para as áreas de produção da Usina Presidente Vargas (UPV), utilizando como insumos água e combustíveis siderúrgicos (gás de alto forno (GAF), gás de aciaria (GLD) e gás de coqueria (GCO) e combustíveis externos de alto valor agregado (Gás Natural (GN) e Óleo BPF (apenas emergências)).

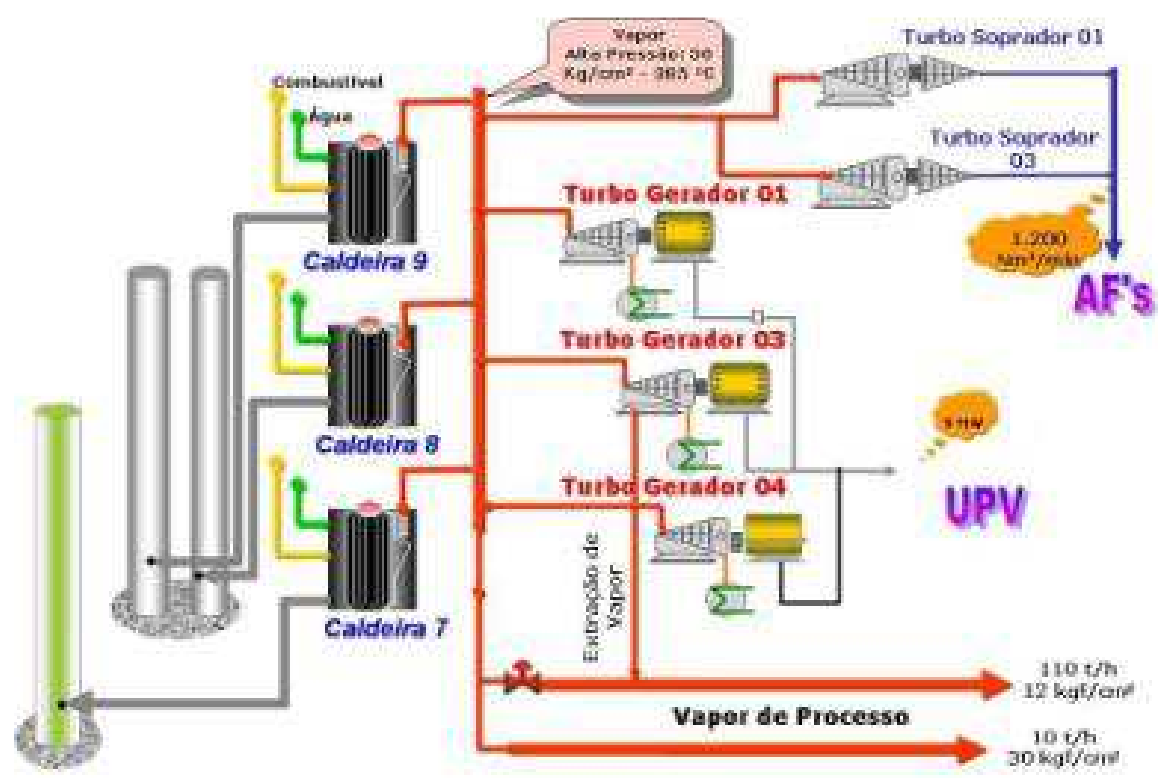

Figura 1. Fluxograma da unidade geradora de energéticos da CTE 1.

Este trabalho tem como objetivo apresentar a implementação do novo modo operacional da CTE-1, com impacto na redução do custo dos produtos (energia elétrica 50hz, vapor de processo) para a Usina Presidente Vargas.

A CTE 1 entrou em operação em 1941 com a geração de 30 MW para atendimento das necessidades de energia da implantação, ao longo dos tempos e das expansões passou a ser considerada como área de emergência para os principais equipamentos da UPV.

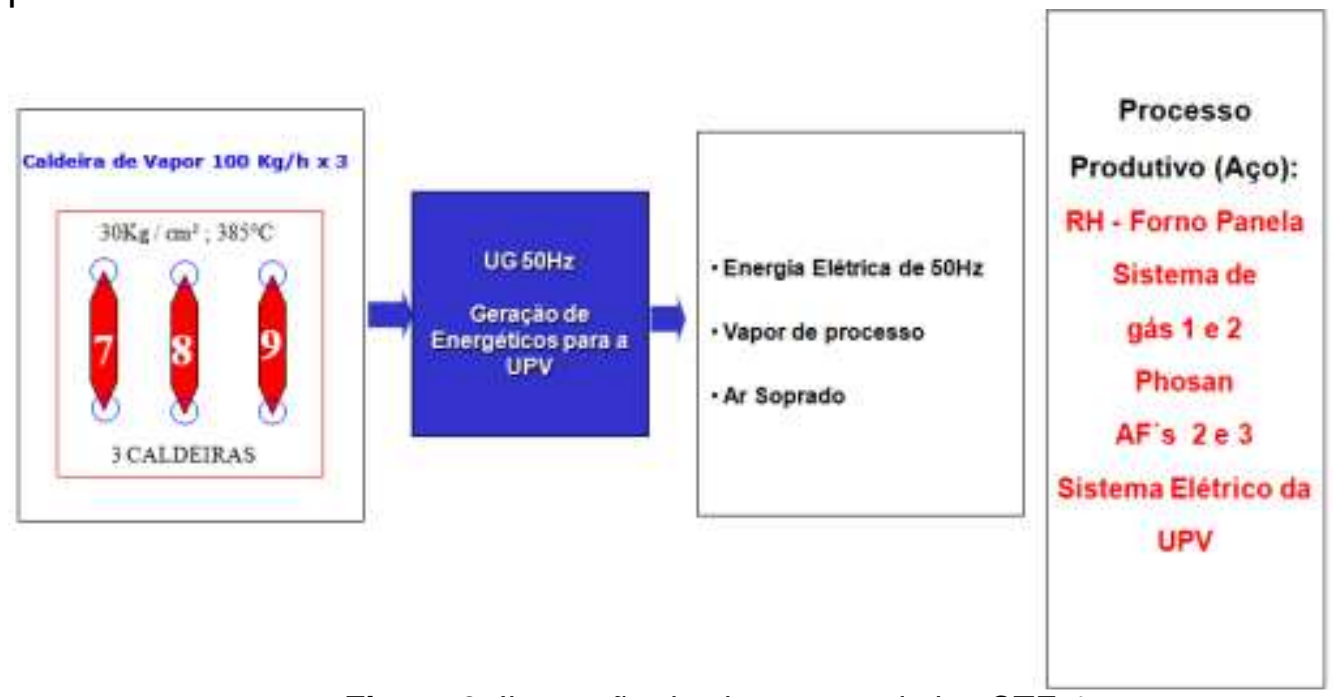

Figura 2. Ilustração do sistema produtivo CTE-1 


\subsection{Descrição do Equipamento da CTE-1}

A CTE-1 tem uma capacidade de geração máxima de $30 \mathrm{MW} / \mathrm{h}$ para abastecer o sistema de geração de emergência da UPV, mostrado abaixo na Figura 3, têm as seguintes características:

- 03 Turbo-Geradores: $30 \mathrm{MW}-13,8 \mathrm{KV}-50 \mathrm{~Hz}$ (cada). GE

- 02 Turbo-Sopradores: $5 \mathrm{MW}$ - INGERSOLL RAND

- Sistema Tratamento Água p/ Caldeiras (2 x $100 \mathrm{t} / \mathrm{h})$

- Sistemas Automação/Controle/Instrumentação

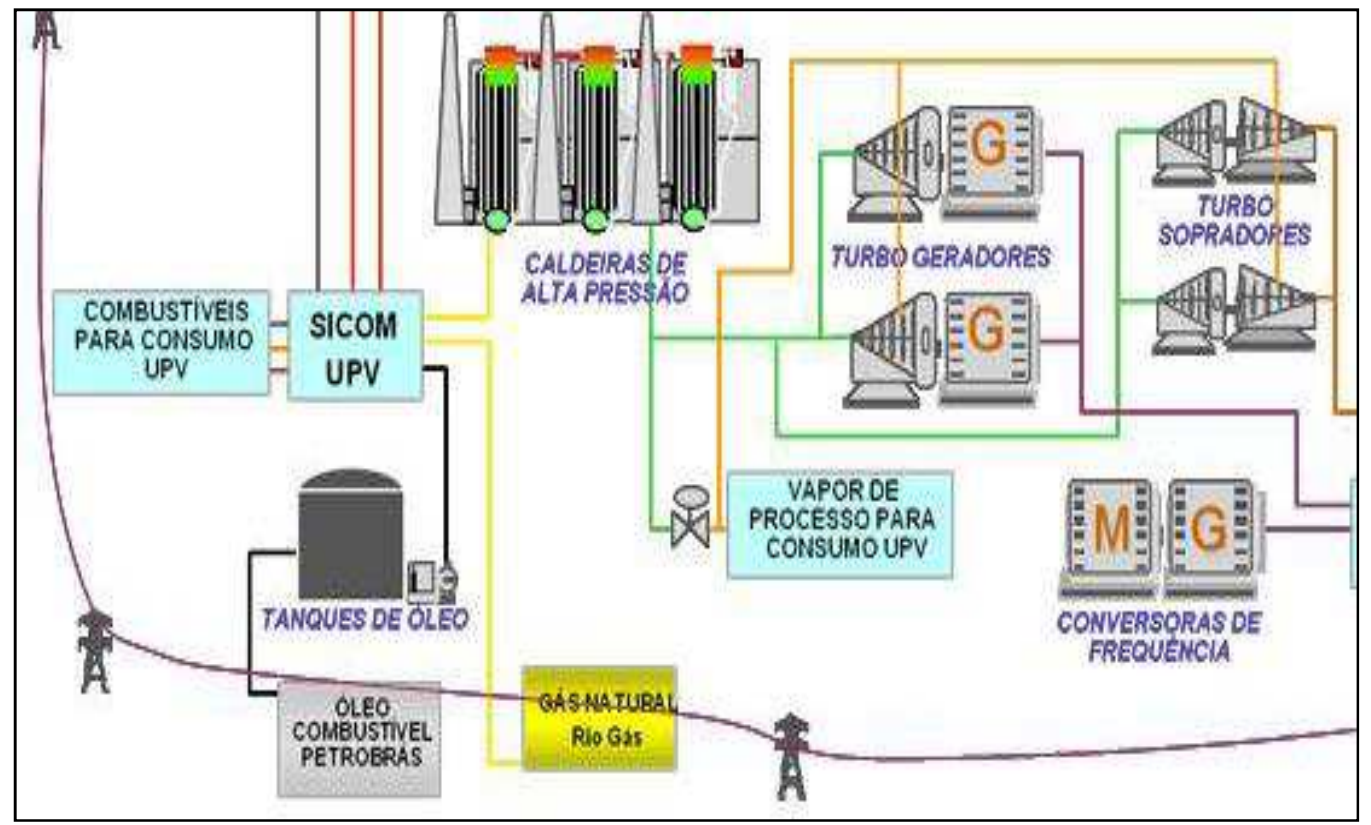

Figura 3. Ilustração do sistema de emergência da UPV

\subsection{Histórico}

Através da avaliação dos cenários atuais de operação do sistema de emergência da UPV e aumento do custo operacional dos produtos vapor e energia elétrica de $50 \mathrm{hz}$ da CTE-1 nos últimos anos (2007-2013), verificou-se a oportunidade de adequação do sistema de geração de vapor e redução de custo para CSN/UPV.

\begin{tabular}{|c|c|c|c|c|c|c|c|}
\hline \multirow[t]{2}{*}{ ITENS } & \multicolumn{6}{|l|}{ REALIZADO } & \multirow{2}{*}{\begin{tabular}{|c|} 
REALIZADO \\
Jul
\end{tabular}} \\
\hline & Jan & Fev & Mar & $\mathrm{Abr}$ & Mai & Jun & \\
\hline E. E. DIST. $50 \mathrm{~Hz}$ (MWh) & 14.614 & 12.599 & 12.937 & 11.913 & 12.484 & 11.909 & 12.546 \\
\hline UG $50 \mathrm{~Hz}$ & $1.065 .411,72$ & $1.126 .684,73$ & $1.318 .778,89$ & $818.141,10$ & $1.121 .347,30$ & $1.115 .991,47$ & $1.251 .351,56$ \\
\hline Energia $60 \mathrm{~Hz}$ para $50 \mathrm{~Hz}$ & $61.593,71$ & $25.503,23$ & $122.511,81$ & $91.633,31$ & $309.838,75$ & $43.109,76$ & $18.189,50$ \\
\hline Custo Distribuiçäo - $50 \mathrm{~Hz}$ & $215.150,81$ & $190.735,07$ & $201.952,01$ & $183.019,91$ & $144.526,91$ & $175.884,98$ & $146.316,42$ \\
\hline TOTAL & $1.342 .229,72$ & $1.342 .923,03$ & $1.643 .242,71$ & $1.092 .794,32$ & $1.575 .712,96$ & $1.334 .986,21$ & $1.415 .857,48$ \\
\hline R\$/MWh & 91,85 & 106,59 & 127,02 & 91,73 & 126,22 & 112,10 & 112,85 \\
\hline
\end{tabular}

Figura 4. Gráfico de custo 2007 de energia elétrica CTE 1.

Para o novo modo operacional da CTE-1, continuaríamos atendendo ao sistema de emergência da UPV. Considerando o estudo realizado da avaliação das novas cargas de energéticos, vislumbrou-se uma oportunidade de ganho aproximado de $\mathrm{R} \$ 5$ milhões/ano. 
Logo o grupo começou a desenvolver uma alternativa para viabilizar a implantação de um novo procedimento de geração de energéticos na CTE-1.

\begin{tabular}{l|r|r|r|r|r|r}
\multirow{2}{*}{ ITENS } & \multicolumn{1}{c}{ REALIZADC } \\
\cline { 2 - 7 } & \multicolumn{1}{|c|}{ jan/13 } & \multicolumn{1}{c|}{ fev/13 } & \multicolumn{1}{c}{ mar/13 } & \multicolumn{1}{c}{ abr/13 } & \multicolumn{1}{c}{ mai/13 } & jun/13 \\
\hline E. ELÉT. UG 50 Hz (MWh) - 7132 & $\mathbf{6 . 0 2 3}$ & $\mathbf{6 . 1 7 1}$ & $\mathbf{6 . 3 4 0}$ & $\mathbf{6 . 0 5 6}$ & $\mathbf{6 . 7 7 0}$ & $\mathbf{6 . 2 8 2}$ \\
\hline Pessoal & $92.053,09$ & $102.554,07$ & $93.038,30$ & $148.045,39$ & $90.879,82$ & $75.748,74$ \\
\hline Outros & $254.499,49$ & $269.135,47$ & $233.723,53$ & $245.258,47$ & $201.875,62$ & $193.761,56$ \\
\hline Água Crua & $138.620,45$ & $143.916,89$ & $214.629,90$ & $192.390,41$ & $240.930,65$ & $218.038,89$ \\
\hline Vapor & $1.777 .667,97$ & $1.563 .166,74$ & $1.153 .004,43$ & $1.344 .118,73$ & $1.371 .125,67$ & $1.467 .703,13$ \\
\hline Manutençäo & $58.399,43$ & $27.942,43$ & $29.475,75$ & $43.488,87$ & $42.576,04$ & $14.844,77$ \\
\hline Depreciaçäo & $46.864,64$ & $46.864,66$ & $46.864,73$ & $46.864,67$ & $46.864,64$ & $46.864,65$ \\
\hline TOTAL & $\mathbf{2 . 3 6 8 . 1 0 5}$ & $\mathbf{2 . 1 5 3 . 5 8 0}$ & 1.770 .737 & $\mathbf{2 . 0 2 0 . 1 6 7}$ & 1.994 .252 & $\mathbf{2 . 0 1 6 . 9 6 2}$ \\
\hline R\$/MWh & 393,18 & 348,98 & 279,30 & 333,58 & 294,57 & 321,07 \\
\hline
\end{tabular}

Figura 5. Gráfico de custo 2013 de energia elétrica CTE 1.

\section{ESTUDO DO CASO}

Com o cenário supracitado, começaram as análises do projeto, verificando os modos de operação e avaliação das condições operacionais.

\subsection{Análise do Projeto}

Com mais de 70 anos de operação, a planta CTE-1 foi projetada para operar com carga de $30 \mathrm{MW} \mathrm{h}$, com uma carga máxima de até $105 \%$. Com 3 caldeiras, com capacidade de carga de 100 ton/h.

O sistema de alta pressão de vapor é operado na pressão de 30 bar e temperatura de $350^{\circ} \mathrm{C}$ com interligação com a CTE-2. O requisito atual de geração para cada TG é de $4 \mathrm{MW}$, para um total de geração de $9 \mathrm{MW}$ h e vapor de processo de $120 \mathrm{ton} / \mathrm{h}$.

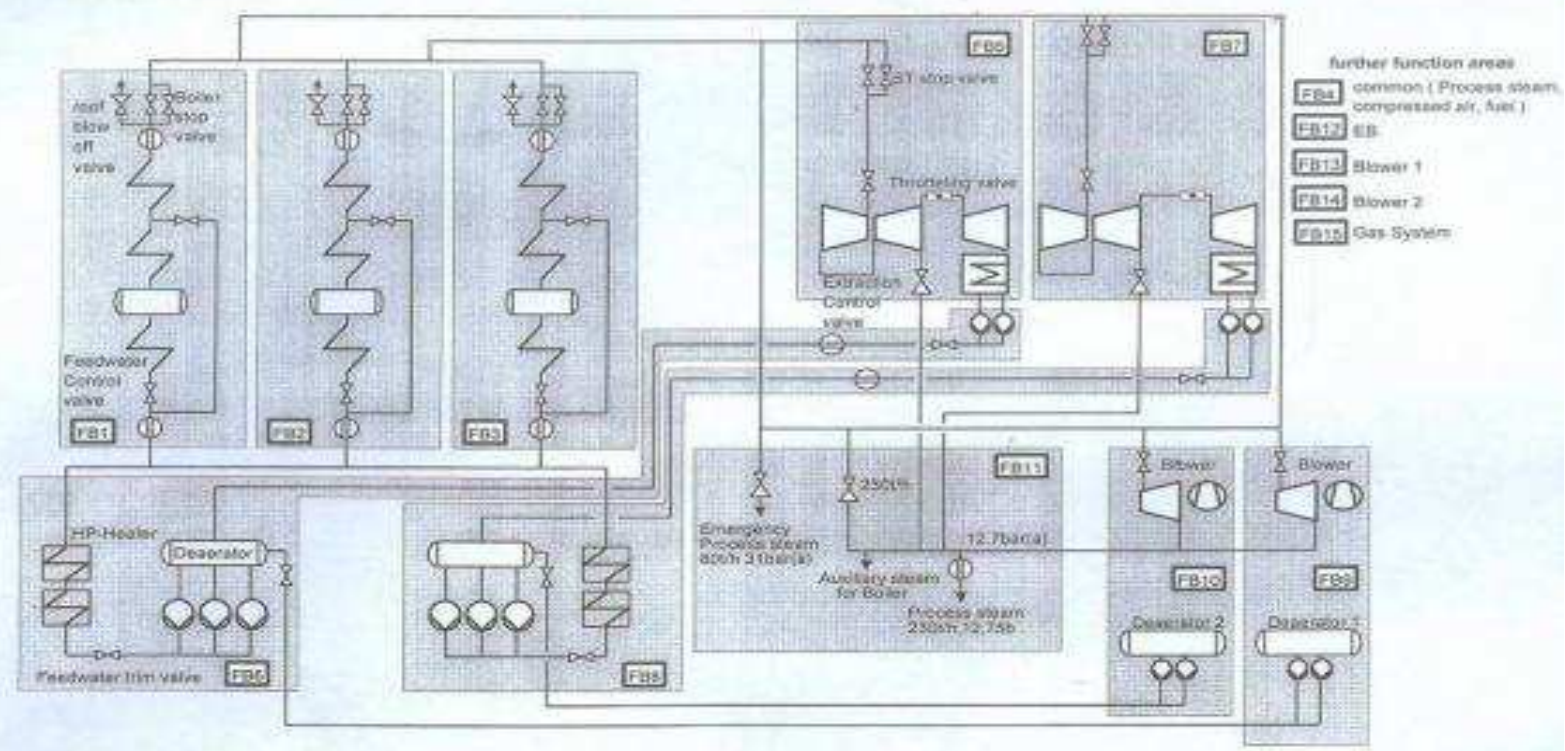

Figura 6. Macro fluxo operacional da CTE-1. 


\subsection{Análise do Processo}

O Processo de energéticos da CTE-1 é formado pela geração dos produtos: ar soprado de emergência para os Altos Fornos, vapor de processo UPV/RH 12bar, vapor de processo 30 bar e energia elétrica. Para geração destes, se faz necessário a absorção de uma grande quantidade de energia na forma de combustíveis internos (GAF, GLD, GCO) e externos de alto valor agregado (GN e ÓLEO). A eficiência da planta é avaliada pela quantidade de energia em gcal dos produtos dividido pela quantidade de energia em gcal dos combustíveis consumidos. Com a sinergia com a CTE-2, a adequação da geração de vapor adicional não afetaria as metas já assumidas e sim benefícios aos equipamentos da geração de energéticos, que se encontra com mais de 50 anos de operação e reduzido nível de eficiência e confiabilidade para os padrões atuais.

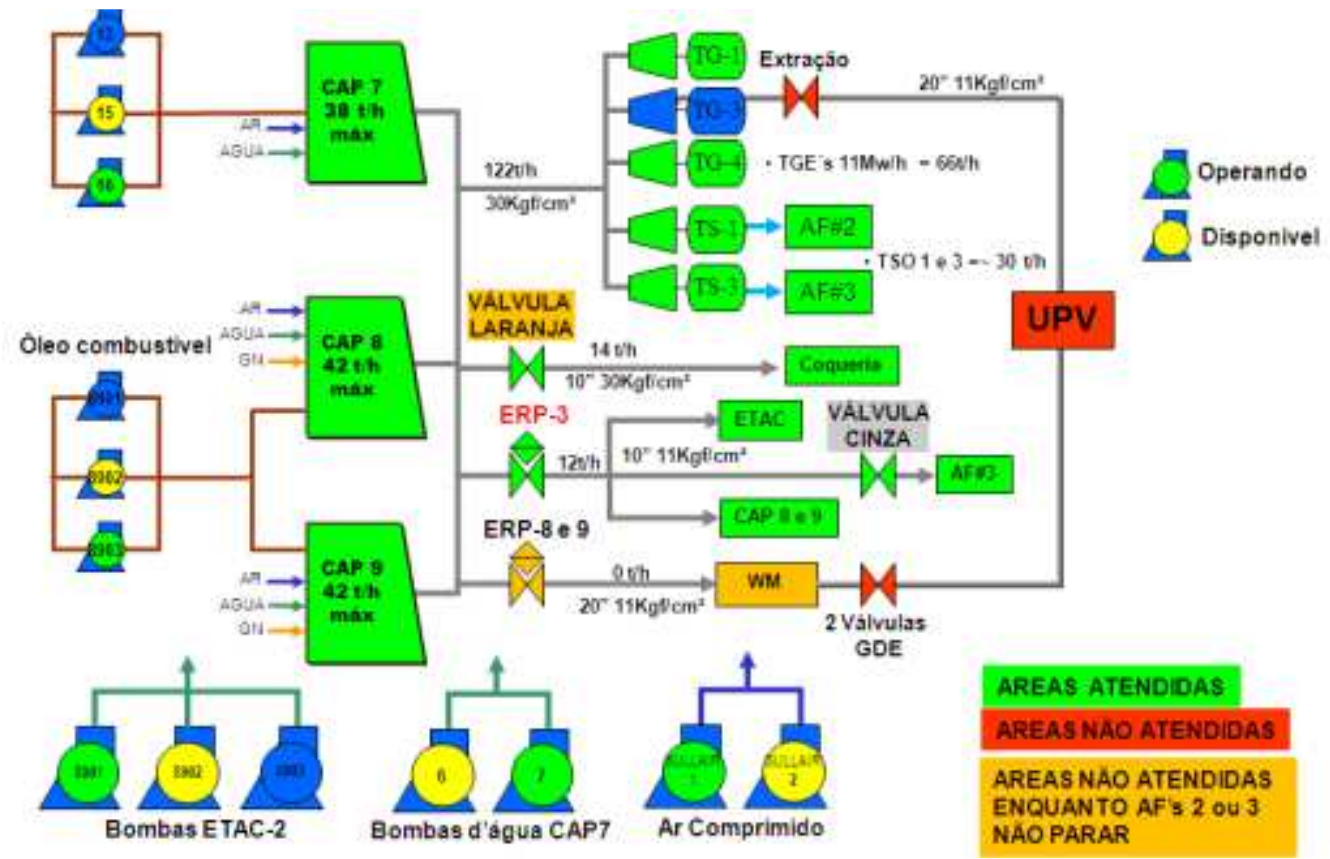

Figura 7. Gráfico de análise de processo da CTE-1.

\subsection{Análise do Consumo e Custo dos Combustíveis}

Para a geração de energéticos da CTE-1, faz necessária a absorção de uma grande quantidade de energia na forma de combustíveis internos (GAF, GLD, GCO) e externos (GN e ÓLEO). Óleo BPF e gás natural são combustíveis externo de alto valor agregado. $\mathrm{Em}$ análise, foi calculado o consumo específico da geração, e através do preço unitário histórico de gás natural, foi possível definir um valor médio, dividindo o preço médio 828,70 reais pelo consumo específico 1,9 obteve o custo de geração de energia elétrica com gás natural na CTE-1 de 350,00 reais por Mwh.

Cenário semelhante para o óleo, devido ao seu poder calorífico de valor aproximado ao gás natural. Conforme análise apresentada fica evidente que a geração de vapor e energia elétrica com acréscimo de gás natural e óleo, mesmo nas situações críticas, afeta diretamente o custo dos produtos da CTE-1. 


\begin{tabular}{c|c|c|c|c|c|} 
& volume & energia & $\begin{array}{c}\text { Eficiência } \\
\text { CTE-1 }\end{array}$ & $\begin{array}{c}\text { Energia } \\
\text { Efetiva }\end{array}$ & Consumo Específico \\
\cline { 1 - 1 } GN & $1 \mathrm{dam}^{3}$ & $8,60 \mathrm{gcal}$ & \multirow{2}{*}{$19 \%$} & $1,63 \mathrm{gcal}$ & \multirow{2}{*}{$1,90 \mathrm{MWh} / \mathrm{dam}^{3}$} \\
\cline { 1 - 3 } & $1 \mathrm{MWh}$ & $0,86 \mathrm{gcal}$ & & $0,86 \mathrm{gcal}$ & \\
\hline
\end{tabular}

Figura 8. Cálculo do consumo específico

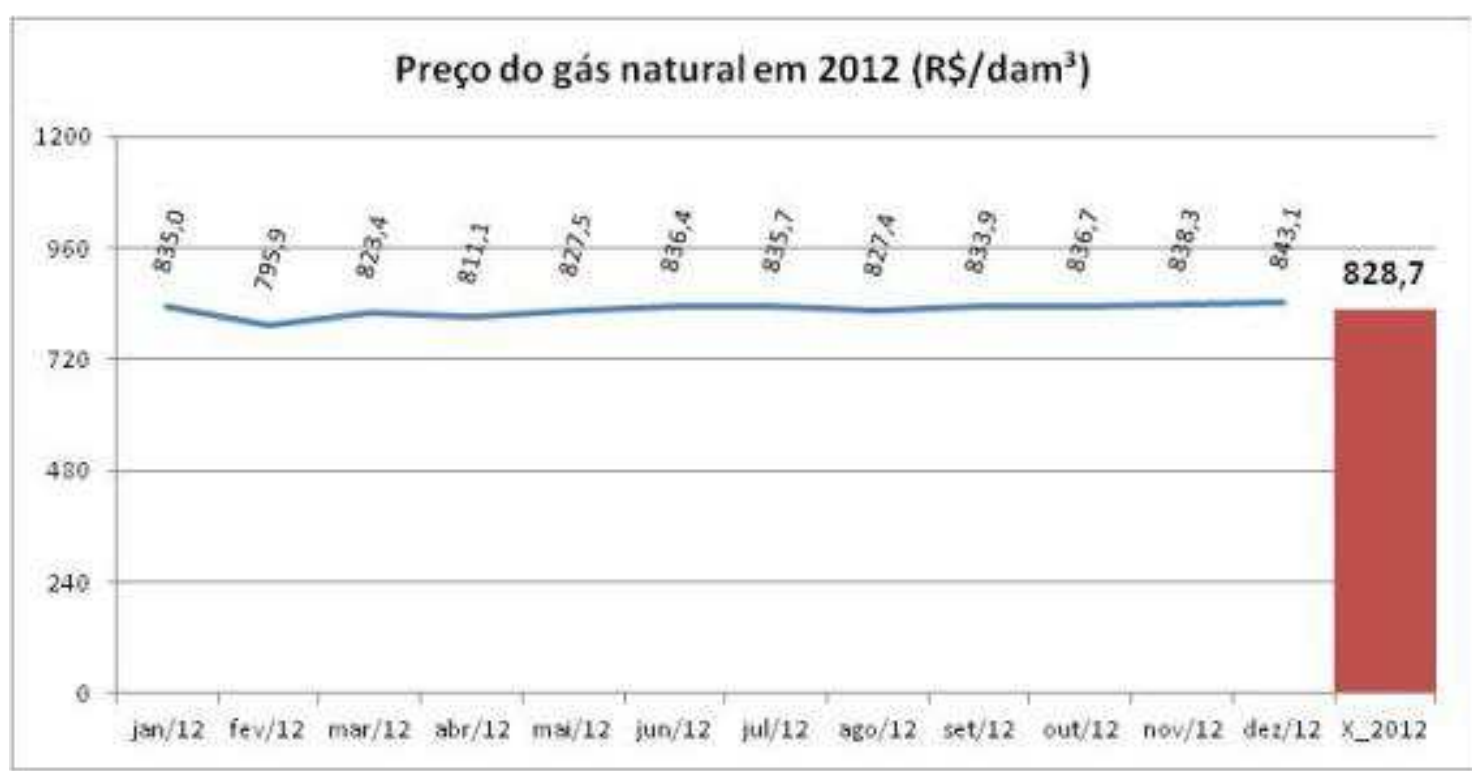

Figura 9. Preço histórico do gás natural

Em julho de 2012, após análise no consumo $X$ custo, vislumbramos a possibilidade de redução no consumo de óleo combustível na CAP\#7.

\section{Custo do Óleo Combustível x Mês - CAP 7 - CTE 1}

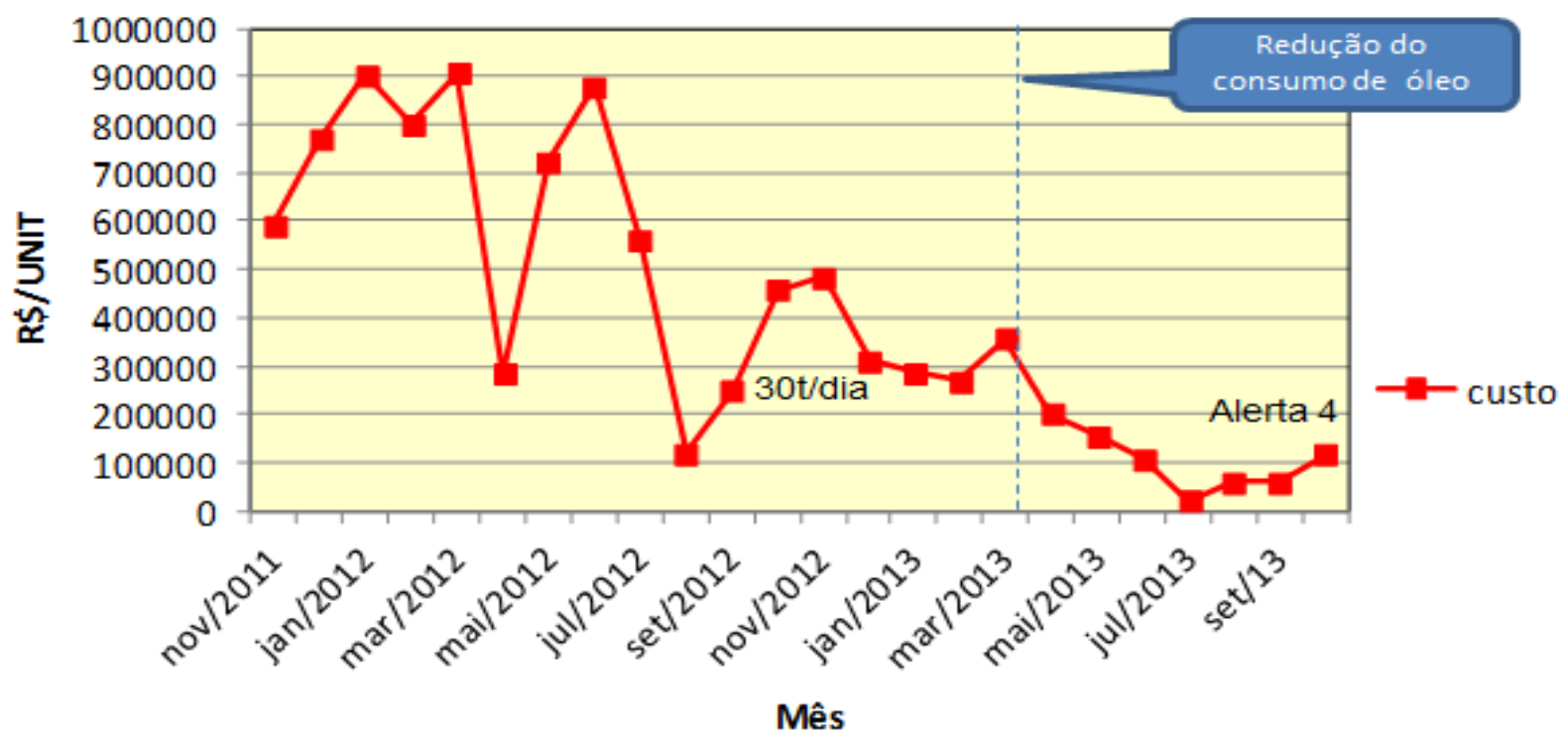

Figura 10. Perfil de consumo de óleo da CAP\#7. 


\subsection{Análise das Cargas Térmicas e Energia de $50 \mathrm{~Hz}$ para UPV}

O sistema de emergência de energia elétrica de $50 \mathrm{~Hz}$ para UPV continua ativo e com grande importância para segurança dos equipamentos, porém o seu conceito é sempre avaliado e adequado às novas cargas e necessidades.

Hoje estamos trabalhando um novo conceito, que a descentralização do sistema de emergência e a sinergia com a CTE-2, que foi projetada para absorver o sistema da UPV.

Assim identificamos a oportunidade de redução das cargas de energia de $50 \mathrm{~Hz}$ de $13 \mathrm{MWh}$ para $9 \mathrm{MWh}$ e redução da geração de vapor na CTE 1 de 180 para 120 ton/h. Esta condição nos favorece para o descomissionamento de alguns sistemas/equipamentos (Caldeira 7 e Turbo Gerador 1), com vida útil desatualizada e baixa eficiência energética.

\begin{tabular}{|c|c|c|c|}
\hline \multirow[t]{2}{*}{ CARGA } & \multirow[t]{2}{*}{ TIPO } & DEMANDA & DEMANDA NA \\
\hline & & MÉDIA [ MW ] & EMERGÊNCIA [ MW] \\
\hline CTE-1 & Prioritária & 0,8 & 0,8 \\
\hline CAC e ECA 1 & Prioritária & 3,2 & 3,2 \\
\hline CBR1 & Prioritária & 1,3 & 1,3 \\
\hline CALDEIRAS 8 e 9 & Prioritária & 1,5 & 1,5 \\
\hline CTE-1 - GMG-230VCC & Prioritária & 0,8 & 0,8 \\
\hline BOMBA LICOR AMONIACAL & Prioritária & 1,9 & 0.4 \\
\hline EMERGÊNCIA AF- 2 & Emergência & 0 & 0.4 \\
\hline EMERGÉNCIA PARA MOTOSOPRADOR & Emergência & 0 & 0,3 \\
\hline EMERGÊNCIA FOXE ESC.CENTRAL & Emergência & 0 & 1.5 \\
\hline EMERG.ACIARIA/LINGOTAMENTO/LTQ2 & Emergência & 0.3 & 3 \\
\hline TOTAL & & 9,8 & 13,2 \\
\hline
\end{tabular}

Figura 11. Perfil de carga atualizado de $50 \mathrm{~Hz}$.

Após análise do sistema de energia da CTE\#1, foi efetuado o descomissionamento do TGE\#1 em agosto de 2013, evitando custo para realização de RG, reduzindo os gastos com manutenção, disponibilizando vapor para o processo.

Aumentando também a segurança operacional e pessoal, sem prejudicar o atendimento da demanda atual.

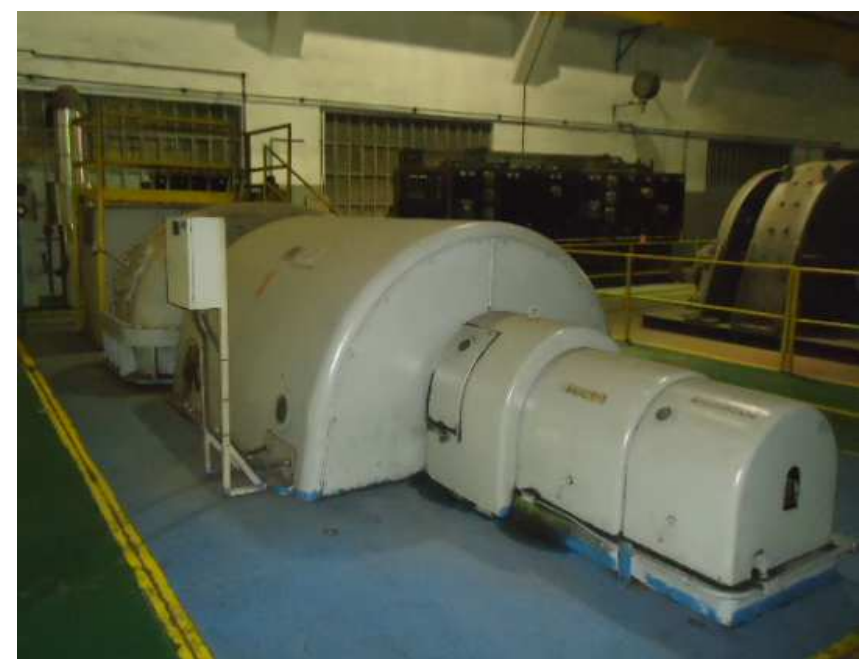

Figura 12. TGE\#1 da CTE\#1 
Mediante também tal análise e devido a redução da geração de vapor pela CTE\#1 em janeiro de 2014, foi efetuado o descomissionamento da CALDEIRA\#7, evitando custo para realização de reparos para atendimento da NR\#13, reduzindo os gastos com manutenção.

Aumentando também a segurança operacional e pessoal, sem prejudicar o atendimento a demanda atual.

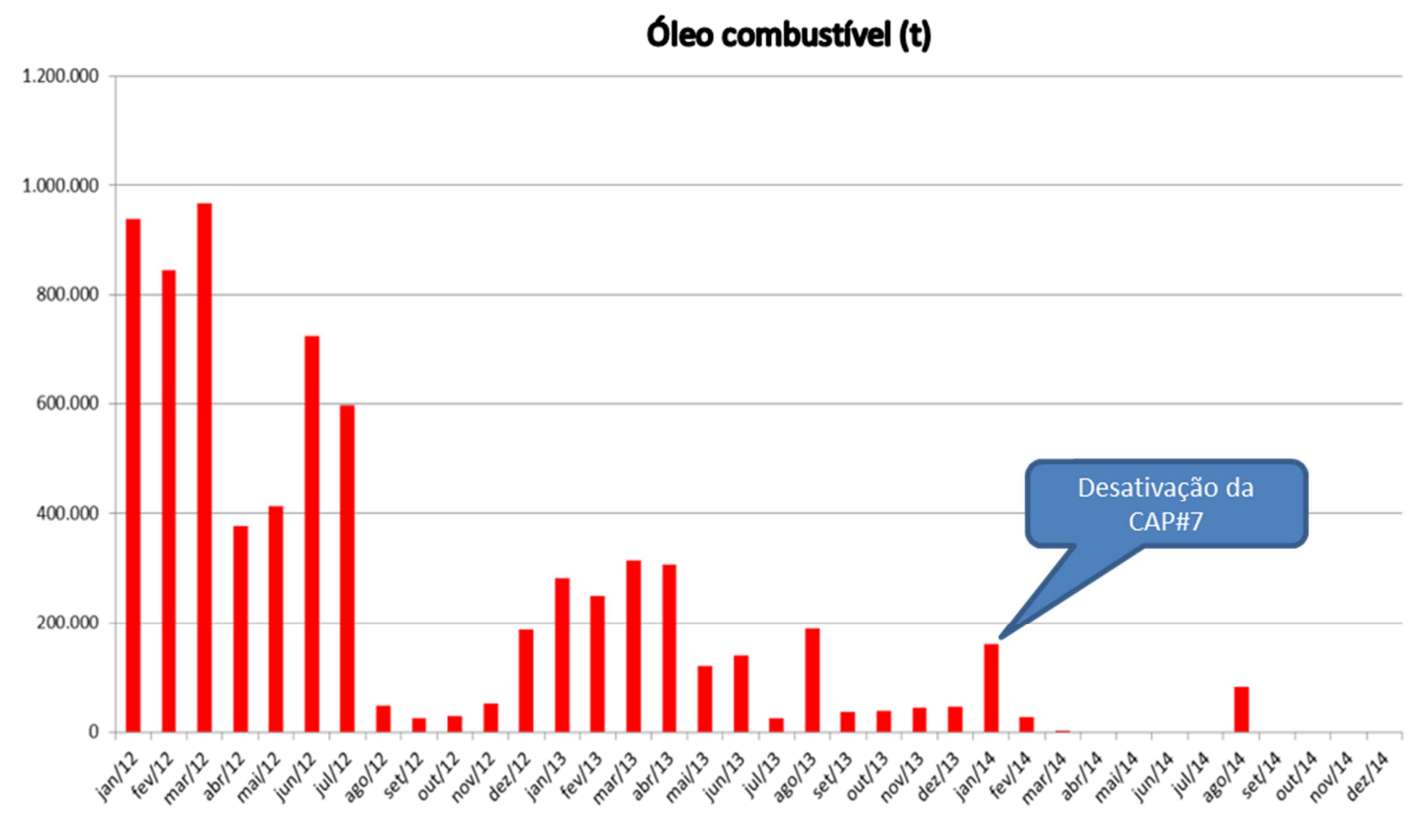

Figura 13. Perfil de consumo de óleo da CTE\#1 após parada da CALDEIRA\#7.

Buscando reduzir o custo total da CTE\#1 e sabendo que os turbo sopradores da CTE-1 eram utilizados como redundância do sistema de emergência (operavam somente na falta da CTE-2 e Moto Sopradores simultaneamente), os mesmos também foram descomissionados em Julho de 2014.
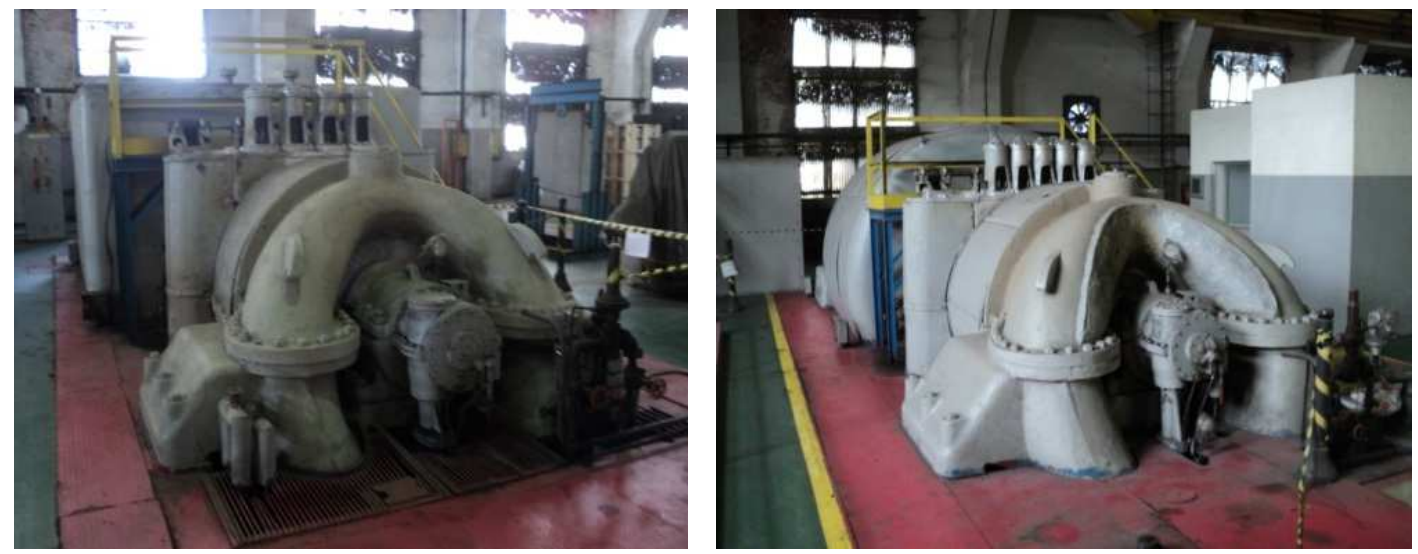

Figura 14. TSO's 1 e 3 da CTE\#1 (respectivamente).

Também em julho de 2014, após análise das cargas térmicas, foi vislumbrado um novo modo de operação das caldeiras 8 e 9 afim de reduzir o custo da produção de vapor. 


\section{$\operatorname{Dam}^{3} \mathrm{GN}$}

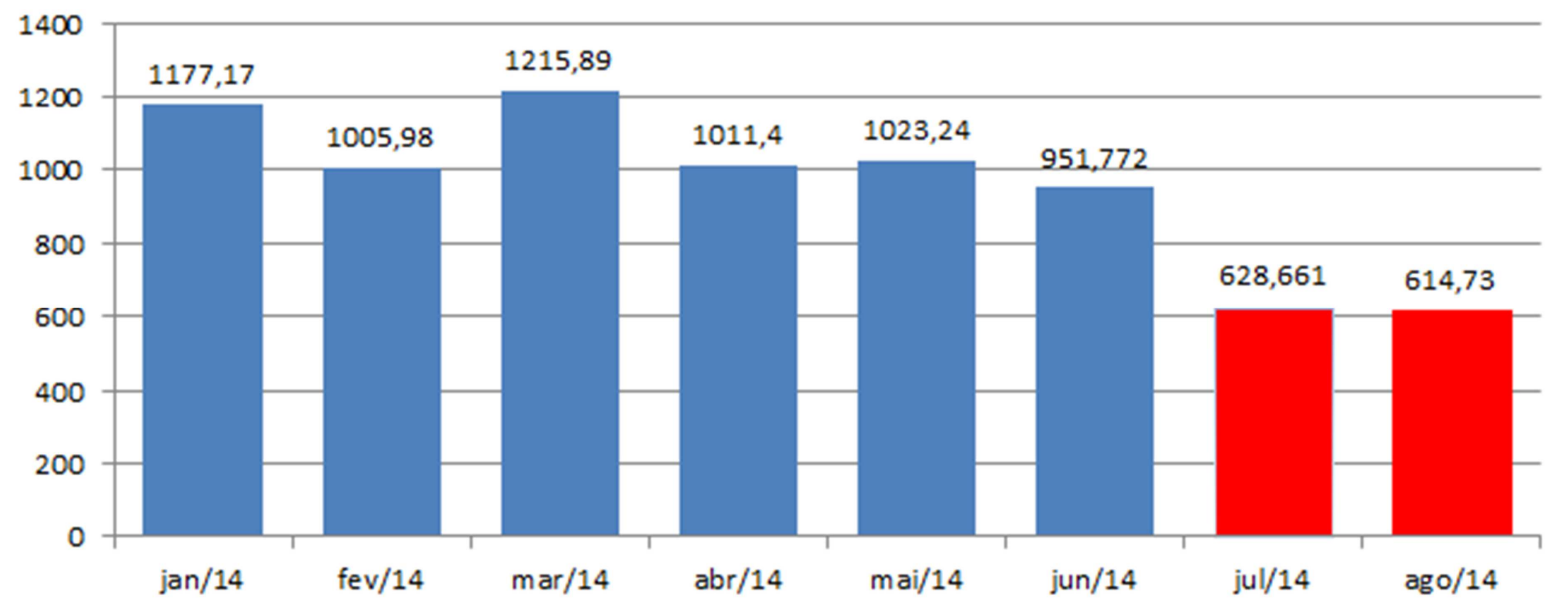

Figura 15. Perfil de consumo de GN nas CALDEIRAS 8 e 9.

\section{RESULTADOS E DISCUSSÃO}

Análise dos resultados dos exames e testes, foram executados seguindo os critérios técnicos operacionais nos principais conceitos empregados, foco nos custos competitivos e condições técnicas dos equipamentos (comportamento da condição operacional). Então, podemos afirmar que a adequação dos procedimentos e geração de energéticos (vapor e geração de energia elétrica) é viável e tem um expressivo retorno monetário para a UPV.

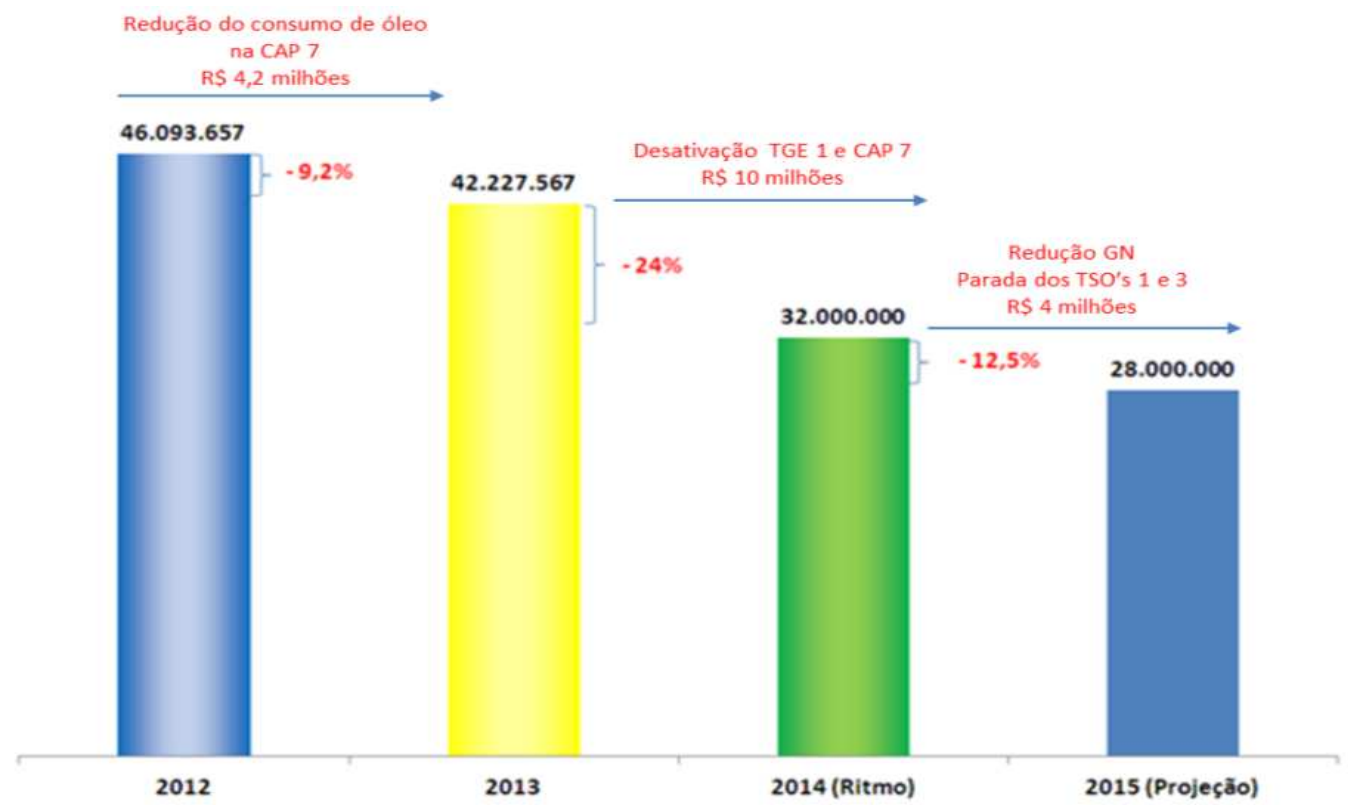

Figura 16. Custo total da CTE\#1.

As avaliações executadas e os novos procedimentos adotados se mostraram efetivos, uma vez que houve um retorno monetário de 4,2 milhões de reais/ano, levando a:

-Redução do consumo de vapor e combustíveis na CTE-1;

-Redução do custo total da CTE-1. 


\section{CONCLUSÃO}

O trabalho mostrou que a metodologia e as ações adotadas pelo grupo foram eficazes, pois com o desenvolvimento dos estudos conseguiu- se interpretar o novo conceito da CTE\#1, adequar às novas cargas de geração de $50 \mathrm{~Hz}$, analisar o processo de geração, diagnosticar e definir a melhor configuração operacional.

Os resultados apresentados foram significativos para as condições de adequações do sistema de vapor, o que representa redução do custo total da CTE 1 e equilíbrio da segurança operacional da planta.

\section{BIBLIOGRAFIA}

1 TORREIRA, Raul Paragallo. GERADORES DE VAPOR / Companhia Melhoramentos, 1995.

2 Manual de Instalação e Operação CBC INDÚSTRIA PESADA - Caldeiras VU-60 da Central Termoelétrica 1, Volta Redonda, 1978.

3 WOODRUFF, Everett B. Steam Plant Operation; Seventh Edition; Hebert B. Lammers; Thomas F. Lammers ; Mc Graw - Hill , 1998.

4 TELLES, Pedro Carlos da Silva. Tubulações Industriais; Rio de Janeiro, Livros Técnicos e Científicos, 1987.

5 API Risk Based Inspection 581, American Petroleum Institute, 1st edition May, 2005.

6 ASME - American Society of Mechanical Engineers - SECTIONS I-Rules for Construction of Power Boiler, Edition 1995. 